\title{
Stationary Wavelet-based Two-directional Two-dimensional Principal Component Analysis for EMG Signal Classification
}

\author{
Yi Ji ${ }^{1}$, Shanlin $\mathrm{Sun}^{2}$, Hong-Bo Xie ${ }^{3}$ \\ ${ }^{I}$ School of Electrical \& Information Engineering, Jiangsu University, Zhenjiang 212013, China \\ ${ }^{2}$ School of Instrumentation Science \& Opto-Electronics Engineering, Beihang University, Beijing 100191, China \\ ${ }^{3}$ ARC Centre of Excellence for Mathematical \& Statistical Frontiers, Queensland University of Technology, Brisbane 4001, \\ Australia,hongbo.xie@qut.edu.au
}

\begin{abstract}
Discrete wavelet transform (WT) followed by principal component analysis (PCA) has been a powerful approach for the analysis of biomedical signals. Wavelet coefficients at various scales and channels were usually transformed into a one-dimensional array, causing issues such as the curse of dimensionality dilemma and small sample size problem. In addition, lack of time-shift invariance of WT coefficients can be modeled as noise and degrades the classifier performance. In this study, we present a stationary wavelet-based twodirectional two-dimensional principal component analysis (SW2D ${ }^{2} \mathrm{PCA}$ ) method for the efficient and effective extraction of essential feature information from signals. Time-invariant multi-scale matrices are constructed in the first step. The two-directional two-dimensional principal component analysis then operates on the multi-scale matrices to reduce the dimension, rather than vectors in conventional PCA. Results are presented from an experiment to classify eight hand motions using 4-channel electromyographic (EMG) signals recorded in healthy subjects and amputees, which illustrates the efficiency and effectiveness of the proposed method for biomedical signal analysis.
\end{abstract}

Keywords: Wavelet transform, principal component analysis, feature extraction, pattern classification, electromyographic signal.

\section{INTRODUCTION}

Biomedical signal analysis has been broadly applied for robotics control, human/brain machine interface, disease diagnosis, wearable devices, and rehabilitation programming. Most biomedical signals, for example electromyography (EMG), an electrical manifestation of skeletal muscle contractions, are typically nonlinear and nonstationary. Discrete wavelet transform (WT) offers simultaneous interpretation of the EMG signal in both time and frequency domains which allows to elucidate local, transient or intermittent components at various scales [1]. However, there are typically a large amount of wavelet coefficients generated from such two-dimensional time-frequency (TF) analysis. In addition to noise interferences, irrelevant or redundant information may exist in the wavelet coefficients. Principal component analysis (PCA) decomposes the covariance structure of the dependent variables into orthogonal components by calculating the eigenvalues and eigenvectors of the data covariance matrix. It linearly projects the original data in a high-dimensional space to a set of uncorrelated components in a low-dimensional feature space while preserving the most original information at the same time. Therefore, WT combined with PCA (WTPCA) has been one of the most powerful approaches to simultaneously extract discriminative feature and reduce the dimension in the EMG study. The basic routine of this hybrid method consists of decomposition of EMG signals into time-frequency plane, rearrangement of the time-frequency elements into a row vector, and reduction of the dimension using PCA. Englehart et al. [2] decomposed four channel transient EMG signals by short-time Fourier transform (STFT), WT, and wavelet packet transform (WPT) into TF plane to discriminate six hand motions for prosthetic hand control. They compared the performance of PCA feature reduction and Euclidean distance class separability (CS) criterion. The results indicated that PCA was vastly superior to $\mathrm{CS}$ dimensionality reduction, as well as significantly improving the WT and WPT-based methods in comparison with time domain feature when using linear discriminant analysis classifier. Khezri and Jahed's study using adaptive neuro-fuzzy inference system further confirmed the superiority of WT-PCA hybridization in EMG-based hand motion pattern recognition [3]. Qi et al. [4], [5] utilized the principal components of EMG intensity spectra obtained from nonlinearly-scaled wavelets to compare motor unit recruitment patterns during isometric ramp and step muscle contractions, as well as dynamic concentric and eccentric contractions of the human biceps brachii. The same WT-PCA scheme was also employed to discriminate between 
fast and slow muscle fibers [6], to investigate motor unit recruitment patterns between and within muscles of the dysfunction in children and young adults with cerebral palsy [8]. Weiderpass et al. [9] investigated the alternations of thigh and calf muscles recruitment strategies during gait among non-diabetic and diabetic neuropathic patients by using an adaptive optimal kernel time-frequency representation and discrete WT followed by PCA. In all of these WTPCA-based EMG representation and recognition methods, WT coefficients at various scales must be first transformed into a vector. However, concatenating WT coefficients at various scales into a 1D array often leads to a high-dimensional vector space, where it is difficult to evaluate the covariance matrix accurately due to its large size and the relatively small number of training samples. Furthermore, computing the eigenvectors of a large size covariance matrix is very time-consuming, whilst the response time of EMG real-time control systems should not introduce a delay that is perceivable by the user [1].

In fact, a two-dimensional WT coefficient matrix can be regarded as an image. It is thus feasible to apply image processing techniques to indicate the WT coefficient matrix characteristics. Two-dimensional principal component analysis (2DPCA) developed by Yang et al. [10] is a 2D image representation and reduction technique, in which an image matrix does not need to be transformed into a 1D array. Many experimental results have indicated that 2DPCA is computationally more efficient than PCA in the extraction of image features. Although 2DPCA is typically able to obtain higher recognition accuracy than PCA, a vital unresolved problem is that 2DPCA needs many more coefficients for image or TF matrix representation than PCA [11]. Zhang and Zhou [11] indicated that 2DPCA essentially operates along the row direction of the image matrix and, thus, proposed an alternative 2DPCA operating along the column direction. By simultaneously considering the row and column directions, they developed the two-directional triceps muscles [7], as well as quantify dynamic muscle two-dimensional principal component analysis (2D 2 PCA) for a more efficient image representation and recognition.

Another issue of discrete wavelet transform is the lack of time-shift invariance caused by down sampling by two. Since all even-indexed outputs of a half-band filter are discarded, a small shift of the input signal causes a large change in the WT sub-band coefficients. Lack of time-shift invariance of WT coefficients can be modeled as noise and degrades the classifier performance [12]. The stationary wavelet transform (SWT) does not decimate the signal at each stage, as does the standard discrete WT, avoiding the problem of nonlinear distortion of the WT with shifts in the signal.

Inspired by the success of $2 \mathrm{D}^{2} \mathrm{PCA}$ in imaging processing and time-invariant characteristics of SWT, the purpose of this study is to develop an efficient and effective feature extraction method for fully exploiting the time-frequency information of biomedical signals. The size of the SWT covariance matrix is equal to the length/width of timefrequency plane in $2 \mathrm{D}^{2} \mathrm{PCA}$, which is quite smaller than the size of a covariance matrix in PCA. The evaluation of covariance matrix is thus more accurate and the estimation of corresponding eigenvectors is more efficient than PCA. The key idea is that $2 \mathrm{D}^{2} \mathrm{PCA}$ is applied to reduce the dimension of SWT coefficient matrix in a highly efficient manner for pattern classification. The method is, therefore, termed as stationary wavelet-based two-directional two-dimensional principal component analysis (SW2D ${ }^{2} \mathrm{PCA}$ ). To illustrate the efficiency and effectiveness of the proposed method, results are presented on the recognition of eight hand motions from 4-channel EMG signals recorded in both healthy subjects and amputees.

\section{SUBJECT \& METHODS}

\subsection{Stationary wavelet transform}

The wavelet transform of a function $f$, with respect to a given mother wavelet $\psi$, is defined as

$$
w_{s} f(x)=f * \psi_{s}(x)=\frac{1}{s} \int_{-\infty}^{\infty} f(t) \psi\left(\frac{x-t}{s}\right) d t,
$$

where $s$ is the scale factor. Assume $s=2^{j} \quad(j \in R, R$ is the integral set), the dyadic WT can be represented as

$$
\begin{aligned}
& S_{2^{j}} f(n)=\sum_{k \in R} h_{k} S_{2^{j-1}} f\left(n-2^{j-1} k\right), \\
& W_{2^{j}} f(n)=\sum_{k \in R} g_{k} S_{2^{j-1}} f\left(n-2^{j-1} k\right),
\end{aligned}
$$

where $S_{2^{j}}$ is a smoothing operator and $W_{2^{j}} f(n)$ is the WT of the discrete signal $f(n)$, whilst $h_{k}$ and $g_{k}$ are the coefficients of corresponding low-pass and high-pass filters, respectively. The standard discrete wavelet transform decimates the wavelet coefficients at each scale, resulting in the half size of the original series. On the other hand, the stationary wavelet transform pads the corresponding low-pass and high-pass filters with zeros between coefficients at each scale. Two new sequences at sub-band thus have the same size as the original sequence. The major advantage of SWT is the preservation of time information of the original signal sequence at each level, particularly useful for feature extraction and denoising [1].

\section{2. $2 D^{2} P C A$ schematic diagram}

Fig.1. is a schematic diagram of $2 \mathrm{D}^{2} \mathrm{PCA}$. Without loss of generality, we consider an $m$ by $n$ time-frequency matrix (TFM) A obtained from the stationary wavelet decomposition. Let $\mathbf{X} \in i^{n \times q}$ and $\mathbf{Y} \in i^{m \times p}$ be matrices having orthonormal columns $n \times q$ and $m \times p$, respectively. We can simultaneously project $\mathbf{A}$ onto $\mathbf{X}$ to yield the $m \times q$ matrix $\mathbf{B}=\mathbf{A X}$, and onto $\mathbf{Y}$ to yield the $p \times n$ matrix $\mathbf{C}=\mathbf{Y}^{T} \mathbf{A}$. In contrast to conventional PCA for onedimensional array applications, 2D 2 PCA operates on a matrix in both horizontal and vertical directions. The total scatter of the projected samples, a measure of the discriminatory power of a projection matrix, can be characterized by its trace of the covariance matrix of the projected matrix. From this point of 
view, maximization of the generalized total scatter is the criterion adopted to find the optimal projection matrices $\mathbf{X}$ and $\mathbf{Y}$ for row and column directions, respectively:

$$
\begin{gathered}
\begin{aligned}
J(\mathbf{X})= & \operatorname{tr}\left\{E\left[(\mathbf{B}-E(\mathbf{B}))(\mathbf{B}-E(\mathbf{B}))^{T}\right]\right\} \\
= & \operatorname{tr}\left\{E\left[(\mathbf{A X}-E(\mathbf{A} \mathbf{X}))(\mathbf{A X}-E(\mathbf{A} \mathbf{X}))^{T}\right]\right\} \\
& =\operatorname{tr}\left\{\mathbf{X}^{T} E\left[(\mathbf{A}-E(\mathbf{A}))^{T}(\mathbf{A}-E(\mathbf{A})] \mathbf{X}\right\},\right. \\
J(\mathbf{Y})= & \operatorname{tr}\left\{E\left[(\mathbf{C}-E(\mathbf{C}))(\mathbf{C}-E(\mathbf{C}))^{T}\right]\right\} \\
= & \operatorname{tr}\left\{E\left[\left(\mathbf{Y}^{T} \mathbf{A}-E\left(\mathbf{Y}^{T} \mathbf{A}\right)\right)\left(\mathbf{Y}^{T} \mathbf{A}-E\left(\mathbf{Y}^{T} \mathbf{A}\right)\right)^{T}\right]\right\} \\
= & \operatorname{tr}\left\{\mathbf{Y}^{T} E\left[(\mathbf{A}-E(\mathbf{A}))(\mathbf{A}-E(\mathbf{A}))^{T}\right] \mathbf{Y}\right\},
\end{aligned}
\end{gathered}
$$

where $\operatorname{tr}\{\bullet\}$ is the trace.

Considering the $m \times q$ matrix $\mathbf{B}=\mathbf{A X}$ obtained by projecting $\mathbf{A}$ onto $\mathbf{X}$ in (4), the horizontal covariance matrix is denoted by

$$
\mathbf{G}_{h}=E\left[(\mathbf{A}-E(\mathbf{A}))^{T}(\mathbf{A}-E(\mathbf{A}))\right],
$$

which is an $n \times n$ positive semi-definite matrix.

Suppose that the training feature set is $\mathbf{\Omega}=\left(\mathbf{A}_{1}, \mathbf{A}_{2}, \mathrm{~L}, \mathbf{A}_{\mathbf{N}}\right)$ , where each $\mathbf{A}_{i}(i=1,2, \mathrm{~L}, \mathbf{N})$ denotes the $i$ th $m \times n$ timefrequency matrix and $\mathbf{N}$ is the number of training samples. The average TFM is given by

$$
\overline{\mathbf{A}}=\frac{1}{N} \sum_{i=1}^{N} \mathbf{A}_{i}
$$

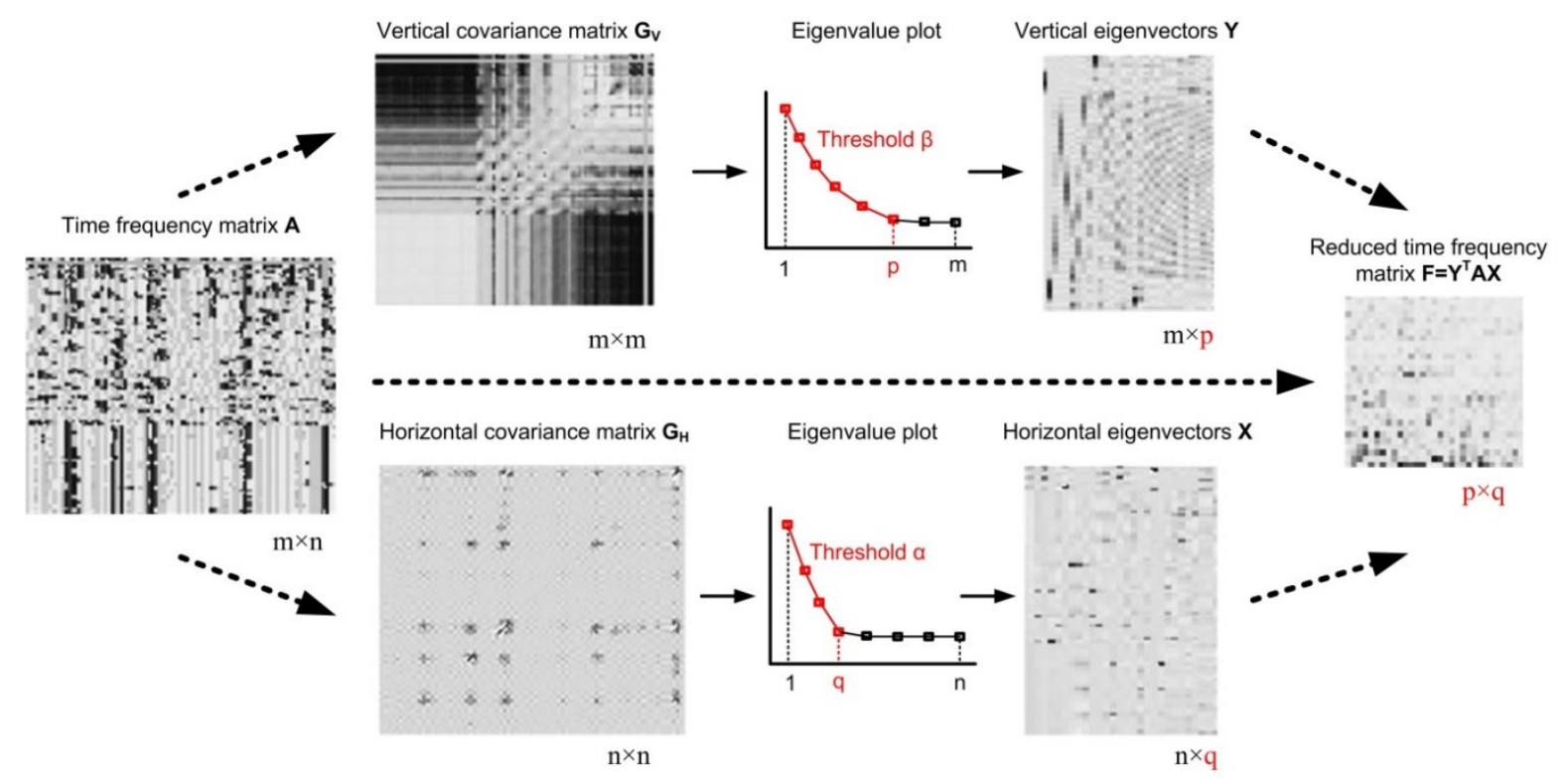

Fig.1. Schematic diagram of two-directional two-dimensional principal component analysis to obtain the reduced time-frequency matrix $\mathrm{F}$

(right) from an input time-frequency matrix A (left).
Denoting the $k$ th row vectors of $\mathbf{A}_{i}$ and $\overline{\mathbf{A}}$ by $\mathbf{A}_{i}^{k}$ and $\overline{\mathbf{A}}_{h}^{k}$, respectively, these TFMs can be represented by

$$
\mathbf{A}_{i}=\left[\left(\mathbf{A}_{i}^{1}\right)^{T},\left(\mathbf{A}_{i}^{2}\right)^{T}, \mathrm{~L},\left(\mathbf{A}_{i}^{m}\right)^{T}\right]^{T},
$$

and

$$
\overline{\mathbf{A}}=\left[\left(\overline{\mathbf{A}}_{h}^{1}\right)^{T},\left(\overline{\mathbf{A}}_{h}^{2}\right)^{T}, \mathrm{~L},\left(\overline{\mathbf{A}}_{h}^{m}\right)^{T}\right]^{T}
$$

The horizontal covariance matrix can then be obtained from the outer product of these TFM row vectors:

$$
\begin{aligned}
\mathbf{G}_{h} & =\frac{1}{N} \sum_{i=1}^{N}\left(\mathbf{A}_{i}-\overline{\mathbf{A}}\right)^{T}\left(\mathbf{A}_{i}-\overline{\mathbf{A}}\right) \\
& =\frac{1}{N} \sum_{i=1}^{N} \sum_{k=1}^{m}\left(\mathbf{A}_{i}^{k}-\overline{\mathbf{A}}_{h}^{k}\right)^{T}\left(\mathbf{A}_{i}^{k}-\overline{\mathbf{A}}_{h}^{k}\right)
\end{aligned}
$$

Similarly, for the $p \times n$ matrix $\mathbf{C}=\mathbf{Y}^{T} \mathbf{A}$ obtained by projecting $\mathbf{A}$ onto $\mathbf{Y}$ in (5), the vertical covariance matrix can be denoted by

$$
\mathbf{G}_{v}=E\left[(\mathbf{A}-E(\mathbf{A}))(\mathbf{A}-E(\mathbf{A}))^{T}\right],
$$

which is $m \times m$ positive semi-definite matrix.

TMFs and their average are now denoted by column vectors:

$$
\begin{gathered}
\mathbf{A}_{i}=\left[\left(\mathbf{A}_{i}^{1}\right)^{T},\left(\mathbf{A}_{i}^{2}\right)^{T}, \mathrm{~L},\left(\mathbf{A}_{i}^{n}\right)\right], \\
\overline{\mathbf{A}}=\left[\left(\overline{\mathbf{A}}_{v}^{1}\right)^{T},\left(\overline{\mathbf{A}}_{v}^{2}\right)^{T}, \mathrm{~L},\left(\overline{\mathbf{A}}_{v}^{n}\right)\right] .
\end{gathered}
$$

where $\mathbf{A}_{i}^{j}$ and $\overline{\mathbf{A}}_{v}^{j}$ denote the $j$ th column vectors of $\mathbf{A}_{i}$ and $\overline{\mathbf{A}}$ , respectively. 
Now, the vertical covariance matrix of (11) can be constructed from the outer products of column vectors:

$$
\begin{aligned}
\mathbf{G}_{v} & =\frac{1}{N} \sum_{i=1}^{N}\left(\mathbf{A}_{i}-\overline{\mathbf{A}}_{h}\right)\left(\mathbf{A}_{i}-\overline{\mathbf{A}}_{h}\right)^{T} \\
& =\frac{1}{N} \sum_{i=1}^{N} \sum_{j=1}^{n}\left(\mathbf{A}_{i}^{j}-\overline{\mathbf{A}}_{v}^{j}\right)\left(\mathbf{A}_{i}^{j}-\overline{\mathbf{A}}_{v}^{j}\right)^{T}
\end{aligned}
$$

Zhang and Zhou [11] demonstrated that the optimal projection matrices $\mathbf{X}$ and $\mathbf{Y}$ are composed of the orthonormal eigenvectors $\mathbf{X}_{1}, \mathbf{X}_{2}, \mathrm{~L}, \mathbf{X}_{q}$ of $\mathbf{G}_{h}$ corresponding to the $q$ largest eigenvalues and $\mathbf{Y}_{1}, \mathbf{Y}_{2}, \mathrm{~L}, \mathbf{Y}_{p}$ of $\mathbf{G}_{v}$ corresponding to the $p$ largest eigenvalues, respectively. The values of $\mathrm{p}$ and $\mathrm{q}$ can be controlled by two pre-set thresholds, and, corresponding to the energy conservation rates at two directions. In practice, we set a ratio of total energy preserved as in PCA (for example $>85 \%$ ) and then set $\alpha=\beta$ [13], [14].

After obtaining the projection matrices $\mathbf{X}$ and $\mathbf{Y}, 2 \mathrm{D}^{2} \mathrm{PCA}$ projects the $m$ by $n$ TFM A onto $\mathbf{X}$ and $\mathbf{Y}$ simultaneously, yielding the reduced $p$ by $q$ matrix

$$
\mathbf{F}=\mathbf{Y}^{T} \mathbf{A} \mathbf{X}
$$

Using the above procedure, an $m \times n$ dimensional feature matrix $\mathbf{A}$ is projected into a $p \times q$ dimensional feature matrix F.

\section{3. $S W 2 D^{2} P C A$ schematic diagram}

In this section, we describe the stationary wavelet-based two directional two-dimensional principal component analysis algorithm for extracting discriminant feature information from these matrices as follows:

1. Multiple-channel signals are first segmented by a moving window with width $d$. Choose a time-frequency decomposition method. That is, specify the mother wavelet function and decomposition level. The stationary wavelet transform is then employed to decompose each time-segment of individual channels into details $D_{1}, D_{2}, \mathrm{~L}, D_{L}$ and approximate $A_{L}$ under the same decomposition level $L$.

2. $2 \mathrm{D}^{2} \mathrm{PCA}$ is subsequently carried out on each of the $d \times(L+1)$ dimension matrices to extract the most informative features, as well as reduce the dimension based on the user-specified threshold of total energy preserved.

3. Since the discriminant abilities of principal components (PCs) at various scales are different, a simple distancebased technique is applied to re-order all PCs [1].

4. The performance of the algorithm is evaluated by feeding the optimal PCs obtained into a classifier.

\subsection{Experimental protocol and performance evaluation}

The proposed algorithm was evaluated using the EMG data collected from the following experiment. Eight distinct wrist and hand motions were used: grasp (GR), hand open (OP), wrist flexion (WF), wrist extension (WE), ulnar deviation (UD), radial deviation (RD), pinch (PN), and thumb flexion (TF), as depicted in Fig.2. These represent the commonly used wrist and hand movements in daily life.

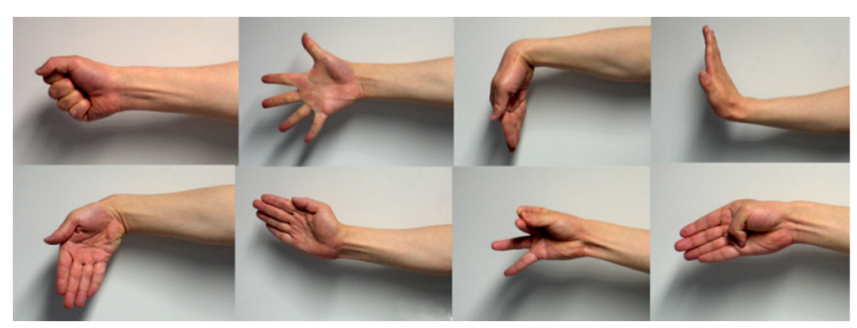

Fig.2. Eight classes of motion were used in the experiment. From the left to right in the first row: grasp (GP), hand open (OP), wrist flexion (WF), wrist extension (WE), and in the second row: ulnar deviation (UD), radial deviation (RD), pinch (PN), thumb flexion (TF).

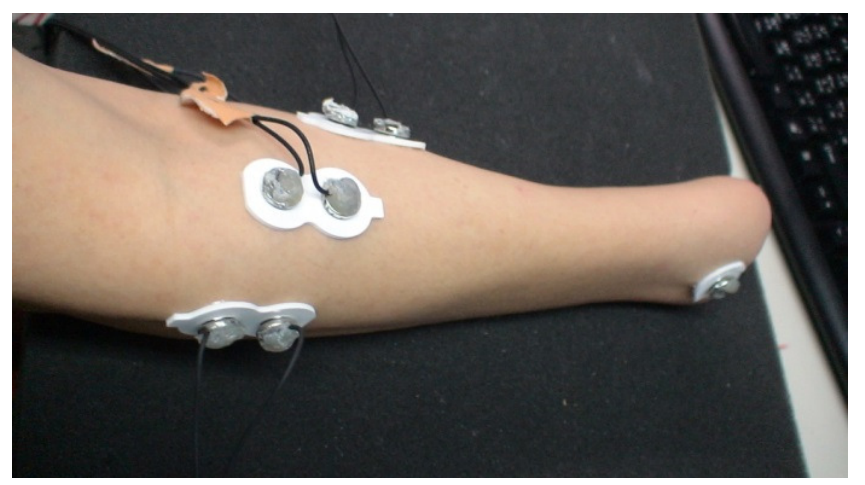

Fig.3. The experimental setting to record EMG signals from an amputee. There are four pairs of electrodes on the forearm with one pair under the forearm. The electrode at the wrist provides the common ground reference.

In the experiment, the EMG data were collected from ten healthy subjects and two amputees (eight males and four females, $30 \pm 6.8$ years). The human subject ethical approval was obtained from the relevant committee and informed consent was obtained from all subjects prior to the experiment. Four channels of EMG signals were acquired from the forearm using the EMG bi-polar $\mathrm{Ag}-\mathrm{AgCl}$ electrodes (Dual electrode \#272, Noraxon USA Inc. AZ, USA). Electrodes were placed on the extensor digitorum, the extensor carpi radialis, the palmaris longus and the flexor carpi ulnaris around the forearm. The distance of two surface electrodes was $2 \mathrm{~cm}$. Skin areas of interest were abrased beforehand with alcohol. An additional $\mathrm{Ag}-\mathrm{AgCl}$ electrode was placed on the wrist to provide a common ground reference. Fig.3. is the experimental setting for an amputee 
with three pairs of electrodes being visible and another pair invisible due to its placement on the other side of the forearm. EMG signal was amplified by an amplifier (RM-6280C, Chengdu Device Inc. Sichuan, China) with a gain of 2000, filtered by $8-500 \mathrm{~Hz}$ band-pass analog filter within the amplifier, and then digitized by a 12-bit data acquisition card (NI PCI-6024E, National Instruments, Austin, TX) with the sample frequency of $1 \mathrm{kHz}$.

Fifteen sessions were conducted for each subject. The first five sessions were used for the learning procedures, while the sixth to tenth session as the validation set and the remaining for performance evaluations. Each subject was asked to maintain a static contraction for each motion and to change the motions with a fixed movement velocity. For those specific tasks the amputees cannot perform, they tried to perform under the guidance. In every session, each motion was performed once for a duration of $5 \mathrm{~s}$, then switched to another motion in random order.

The 4-channel EMG data were further segmented into a series of overlapping windows (window length: $256 \mathrm{~ms}$, overlap step: $128 \mathrm{~ms}$ ). Since the Symmlet-5 has been proven to be an effective mother wavelet for stationary waveletbased classification [15], it was selected to simultaneously decompose EMG signals over six levels. The remaining procedures for SW2D ${ }^{2} \mathrm{PCA}$ described in Section 2.3 were employed to extract two-dimensional PCs. Support vector machine (SVM), a typical nonlinear EMG classifier used in previous study [1]-[3], was employed to evaluate the classification performance of the proposed algorithm. After the classification, the accuracy was further improved by a post-processing procedure using majority vote (MV) [16]. Conventional WTPCA algorithm to analyse the same data set was also devised for comparison.

$\mathrm{CH} 1$

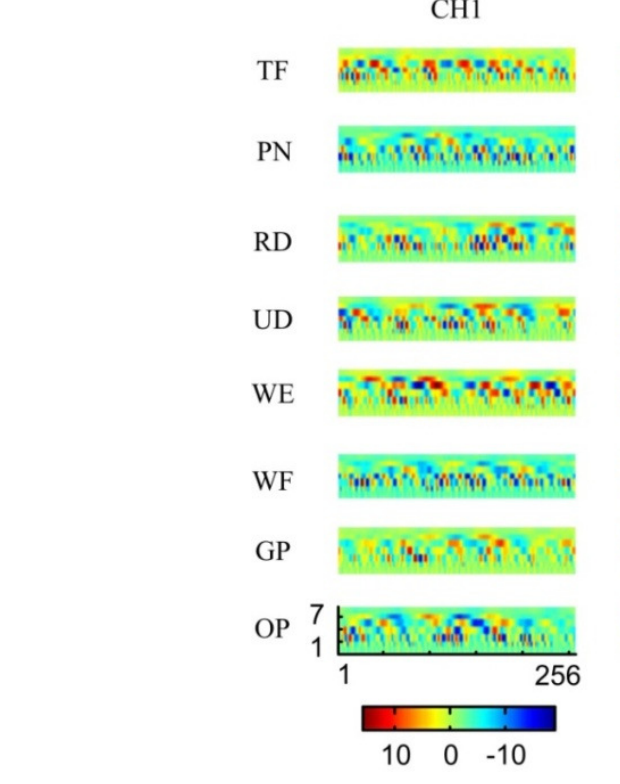

$\mathrm{CH} 2$

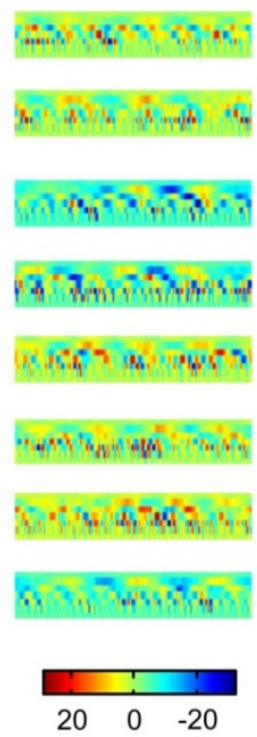

\section{RESULTS}

\subsection{Multi-scale muscle activity patterns}

Using the proposed SW2D 2 PCA technique, the EMG signal at each channel was first transformed into a two-dimensional matrix. Fig.4. shows the typical contour plots for eight motions for subject 3 , each row corresponding to a motion type. With each intended motion, a significant difference between the intensity of the surface EMG signals over the upper limb muscles can be readily discerned in the first column contour plots. Similar to the panels in the first column, there was significant discrepancy in the intensity distributions of the remaining contour plots in the remaining three columns, indicating useful discriminant information in the SWT matrices.

The two-directional two-dimensional principal component analysis was then used to reduce the dimension of each matrix. Fig.5. shows the contour plots of each matrix in Fig. 4. following dimension reduction using $2 \mathrm{D}^{2} \mathrm{PCA}$ when the energy conservation rate and total energy preserved were $98 \%$ and $90 \%$, respectively. Compared with Fig.4., the intensity difference between certain sub-panels in Fig.5. is further enhanced, including, for example, those in the first row. In addition, the matrix size at each channel was significantly decreased, which were $80 \times 3,86 \times 3,122 \times 4$, and $116 \times 4$, respectively. If conventional PCA was used with all time-frequency coefficients arranged into a $1 \mathrm{D}$ array, the size of the covariance matrix would be $1792 \times 1792$. However, the use of $2 \mathrm{D}^{2} \mathrm{PCA}$ resulted in the size of all covariance matrices being less than $130 \times 130$, avoiding the curse of dimensionality and small sample issue as well as improving the numerical stability. It should be noted that the reduced dimension of each channel for all subjects were different because EMG signal varied from subject to subject due to the physiological factors.

Fig.4. Contour plots of stationary wavelet transform matrices for 4-channel EMG traces of eight hand motions obtained from subject 3. The abscissa represents the time and the ordinate represents the frequency or the scale of stationary wavelet transform. The color bar indicates the strength of the muscle electrical activity. 

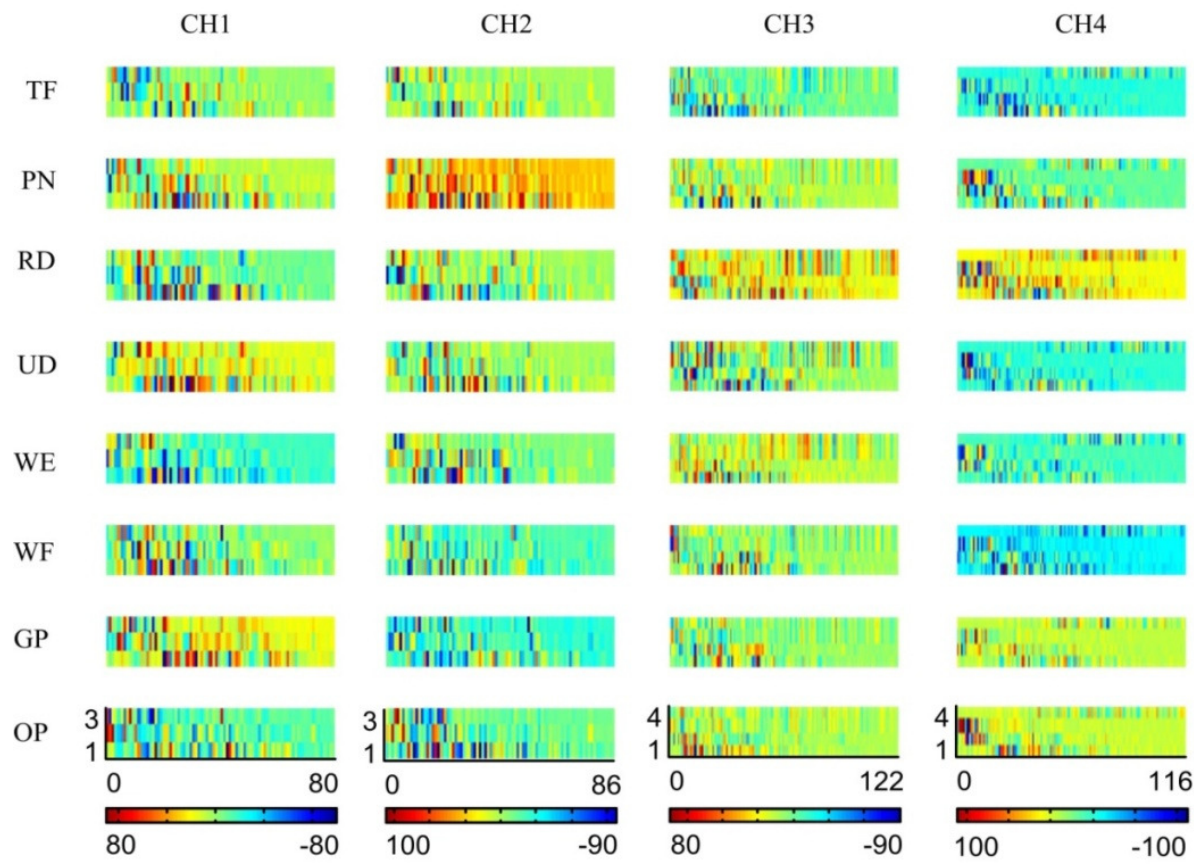

Fig.5. The contour plots of stationary wavelet transform matrices reduced using 2D $2 \mathrm{PCA}$ for 4-channel EMG signals of eight hand motions obtained from subject 3. The abscissa and ordinate represent the reduced size of the contour plots in Fig.4. The color bar indicates the relative strength of the muscle myoelectric activity after dimension reduction.

\subsection{Effect of energy conservation rate}

A large energy conservation rate results in more information loss, whilst a low rate increases the computational burden. To reach a trade-off between these two factors, three energy conservation rates of $97 \%, 98 \%$ and $99 \%$, were employed to assess its effect on classification accuracy. Fig.6. shows the average accuracy across the 12 subjects at these various energy conservation rates for the SVM classifier. With the increasing number of PCs, the accuracy of all three conservation rates initially increased and then entered a relatively flat range with moderate fluctuations. The optimal PCs to achieve the highest accuracy for three conservation rates were all in the range from 25 to 35. In addition, there was no significant difference between energy conservation rates $(p<0.01)$.

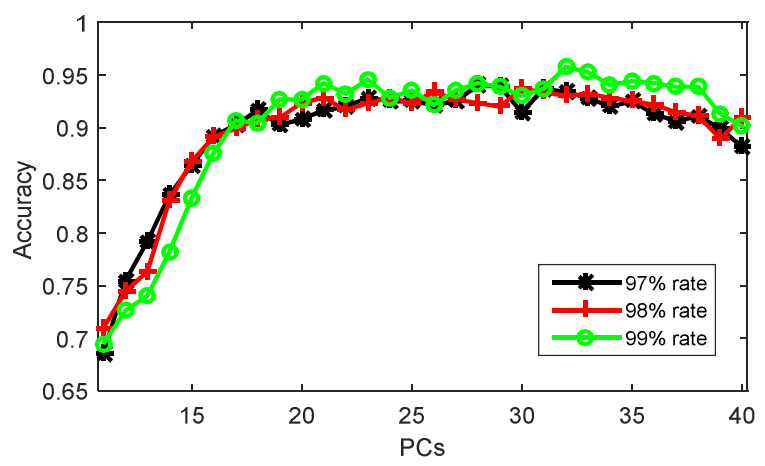

Fig.6. The effect of $97 \%$ (black), $98 \%$ (red), and $99 \%$ (green) energy conservation rate of $\mathrm{SW} 2 \mathrm{D}^{2} \mathrm{PCA}$ on the $\mathrm{EMG}$ signals classification accuracy.

\subsection{Effect of total energy conserved}

For PCA analysis, a typical recommendation is to set the threshold of total energy conserved between 0.8 and 0.95 . Fig.7. shows the classification accuracy for SVM for three threshold values of total energy conserved, i.e., $95 \%, 90 \%$, and $85 \%$. With the reduction in threshold, there was no significant difference in the accuracy for SVM. The insensitivity of SVM to the total energy preserved may be due to its adaptive ability to map input features to highdimensional feature space.

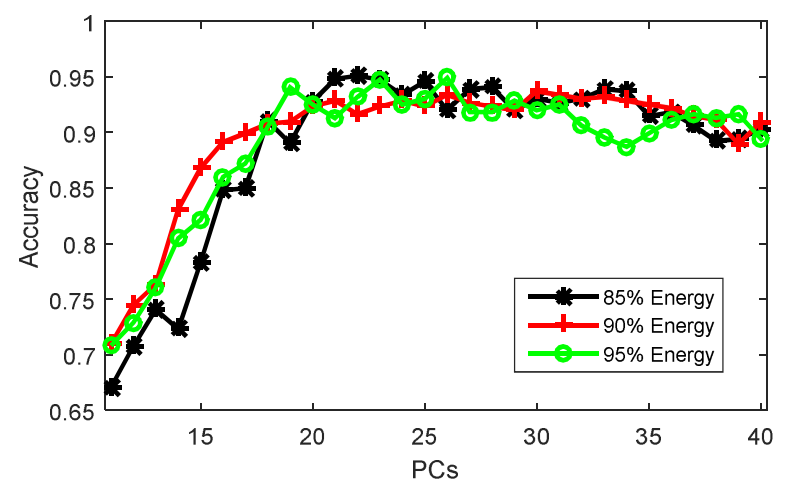

Fig.7. The effect of $85 \%$ (black), $90 \%$ (red), and $95 \%$ (green) total energy conserved of SW2D2PCA on the EMG signals classification accuracy.

\subsection{Recognition of intended motions}

Pattern recognition analysis was performed using the optimal number of PCs previously determined. Table 1. summarizes the subject-specific classification accuracy for 
all eight intended upper-limb motions. An average classification accuracy above $93 \%$ could be achieved among all subjects after majority vote. Across all subjects, there is significant difference between the accuracy of SW2D ${ }^{2} \mathrm{PCA}$ and WTPCA $(p<0.05)$ with lower average accuracy for WTPCA in both cases - with or without majority vote. On the other hand, because the amputees can only perform grasping, opening based on imagination, the related muscle activities were not as strong as in the healthy subjects. The accuracy for two amputees was much lower than for the healthy subjects. As mentioned before, it should be emphasized that EMG activity is subject-dependent for both healthy subjects and amputees. Therefore, the structure and information distribution in the time-frequency matrices varied between subjects, which led to different reduced sizes with SW2D2PCA. Ultimately, this subject-specific time-frequency distribution of EMG feature information led to inconsistent classification errors among different subjects. The subjectspecific EMG activity and classification performance suggested that optimal myoelectric pattern-recognition control system parameters should be individually customized.

Table 1. Classification results of all twelve subjects by proposed stationary wavelet two-directional two-dimensional principal component analysis and conventional wavelet principal component analysis based feature subsets

\begin{tabular}{|c|c|c|c|c|}
\hline \multirow{2}{*}{ Subject } & \multicolumn{2}{|c|}{ Before MV } & \multicolumn{2}{c|}{ After MV } \\
\cline { 2 - 5 } & $\begin{array}{c}\text { SW2D PC } \\
\text { A }\end{array}$ & WTPCA & $\begin{array}{c}\text { SW2D }{ }^{2} \mathrm{PC} \\
\text { A }\end{array}$ & WTPCA \\
\hline 1 & 92.15 & 83.52 & 98.28 & 89.31 \\
\hline 2 & 88.92 & 85.19 & 95.66 & 94.28 \\
\hline 3 & 89.50 & 90.54 & 95.33 & 95.95 \\
\hline 4 & 91.65 & 86.09 & 96.64 & 92.73 \\
\hline 5 & 89.98 & 80.94 & 94.73 & 84.06 \\
\hline 6 & 91.11 & 84.53 & 97.86 & 91.69 \\
\hline 7 & 93.39 & 88.75 & 98.23 & 96.35 \\
\hline 8 & 91.91 & 83.28 & 94.31 & 87.92 \\
\hline 9 & 92.27 & 91.04 & 99.95 & 96.00 \\
\hline 10 & 89.93 & 82.58 & 95.57 & 87.76 \\
\hline $11 *$ & 79.74 & 72.55 & 86.11 & 77.78 \\
\hline $12 *$ & 72.29 & 68.16 & 78.47 & 71.09 \\
\hline Averag & $88.57 \pm 6.2$ & $83.09 \pm 6$. & $94.26 \pm 6.1$ & $88.74 \pm 7$. \\
\hline
\end{tabular}

\section{DiSCUSSION / CONCLUSIONS}

A novel stationary wavelet-based two-directional twodimensional principal component analysis for signal classification has been proposed and examined in this study. One of the major challenges related to the design of EMG interfaces is to maintain high classification accuracy in longterm use [17]. In real use, the muscle contractions, i.e. the classes associated to control commands, are performed in a variety of conditions, which may lead to differences in signal properties making them unrecognizable for the classifier. Stationary wavelet transform avoids the problem of nonlinear distortion of the wavelet and wavelet package transforms with shifts in the signal. Recently, with the improvements in physiological measurement equipment for EMG as well as electroencephalography and magnetoencephalography, new technology permits registration of up to several hundred channels using high-density electrode arrays. Such arrays with small electrode sizes and inter-electrode spacing can cover large areas of the tissue, providing extra spatial information which is largely independent of any "classical" temporal information. To effectively and efficiently extract feature from such high-dimensional signal space is another challenge in biosignals analysis and their applications [18]. Compared with the existing PCA method, 2D²PCA provides an improved approach to extract discriminative feature and reduce the dimension from the high-dimensional random and complex raw signals.

In order to test this approach, we used SW2D ${ }^{2} \mathrm{PCA}$ to extract and classify specific TF patterns in four-channel EMG signals from ten healthy subjects and two amputees for identification of eight hand motions. SW2 ${ }^{2} \mathrm{PCA}$ achieved higher accuracy than WTPCA for both healthy subjects and amputees before and after majority vote. For the healthy subjects, the average accuracy exceeds $96.6 \%$, which can be employed as a promising technique for human-machine interaction or robot control. However, in comparison to the healthy subjects, the classification accuracy for two amputees using SW2D ${ }^{2}$ PCA is relatively low although it is higher than that of WTPCA. This is due to the fact that the amputees can only perform grasping and opening based on imagination, the relevant muscle activities were not as strong as in the healthy subjects. In this study, Symmlet-5 was employed as the mother wavelet to decompose EMG signals for all subjects. Studies have indicated that signal matched or optimized wavelet can substantially enhance the classification accuracy [19], [20]. Another limitation of this study is that the PCs of each channel obtained using 2 $\mathrm{D}^{2} \mathrm{PCA}$ are re-ordered and further reduced using a simple distance measure, which is equivalent to a two-step reduction method. It is necessary to develop a unified framework to simultaneously extract discriminative features from multiple channels. In addition, effective training can improve the electrical activities of residual muscles, and, therefore decrease the recognition error for amputees. It is expected that integration of these measures will result in enhanced pattern recognition of motion patterns for the amputees. The efficiency and effectiveness of the method can be further validated by using high-dimensional EEG, MEG, and fMRI signals. Although the present study focuses on signal pattern classification, based on the PCs obtained from time-frequency plane, it is relatively straightforward to expand $\mathrm{SW} 2 \mathrm{D}^{2} \mathrm{PCA}$ for signal compression, denoising, instantaneous frequency estimation, and other related tasks. 


\section{REFERENCES}

[1] Xie, H.B., Zheng, Y.P., Guo, J.Y. (2009). Classification of the mechanomyogram signal using a wavelet packet transform and singular value decomposition for multifunction prosthesis control. Physiological Measurement, 30, 441-457.

[2] Engelhart, K., Hudgins, B., Parker, P.A., Stevenson, M. (1999). Classification of the myoelectric signal using time-frequency based representations. Medical Engineering \& Physics, 21, 431-438.

[3] Khezri, M., Jahed, M. (2007). Real-time intelligent pattern recognition algorithm for surface EMG signals. Biomedical Engineering Online, 6, 45.

[4] Qi, L.P., Wakeling, J.M., Ferguson-Pell, M. (2011). Spectral properties of electromyographic and mechanomyographic signals during dynamic concentric and eccentric contractions of the human biceps brachii muscle. Journal of Electromyography and Kinesiology, 21, 1056-1063.

[5] Qi, L.P., Wakeling, J.M., Green, A., Lambrecht, K., Ferguson-Pell, M. (2011). Spectral properties of electromyographic and mechanomyographic signals during isometric ramp and step contractions in biceps brachii. Journal of Electromyography and Kinesiology, $21,128-135$

[6] Von Tscharner, V., Goepfert, B. (2006). Estimation of the interplay between groups of fast and slow muscle fibers of the tibialis anterior and gastrocnemius muscle while running. Journal of Electromyography and Kinesiology, 16, 188-197.

[7] Wakeling, J.M., Uehli, K., Rozitis, A.I. (2006). Muscle fibre recruitment can respond to the mechanics of the muscle contraction. Journal of the Royal Society Interface, 3, 533-544.

[8] Wakeling, J., Delaney, R., Dudkiewicz, I. (2007). A method for quantifying dynamic muscle dysfunction in children and young adults with cerebral palsy. Gait \& Posture, 25, 580-589.

[9] Weiderpass, H.A., Pachi, C.G.F., Yamamoto, J.F., Hamamoto, A., Onodera, A.N., Sacco, I.C.N. (2013). Time-frequency analysis methods for detecting effects of diabetic neuropathy. International Journal for Numerical Methods in Biomedical Engineering, 29, 1000-1010.

[10] Yang, J., Zhang, D., Frangi, A.F., Yang, J.Y. (2004). Two-dimensional PCA: A new approach to appearancebased face representation and recognition. IEEE
Transactions on Pattern Analysis and Machine Intelligence, 26, 131-137.

[11] Zhang, D.Q., Zhou, Z.H. (2005). (2D)(2)PCA: Twodirectional two-dimensional PCA for efficient face representation and recognition. Neurocomputing, 69, 224-231.

[12] Kiatpanichagij, K., Afzulpurkar, N. (2009). Use of supervised discretization with PCA in wavelet packet transformation-based surface electromyrogram classification. Biomedical Signal Processing and Control, 4, 127-138.

[13] Jolliffe, I.T., Cadima, J. (2016). Principal component analysis: A review and recent developments. Philosophical Transactions of The Royal Society A Mathematical Physical and Engineering Sciences, 374, 20150202

[14] Bro, R., Smilde, A.K. (2014). Principal component analysis. Analytical Methods, 6, 2812-2831.

[15] Englehart, K., Hudginsa, B., Chan, A.D.C. (2003). Continuous multifunction myoelectric control using pattern recognition. Technology and Disability, 15, 95103.

[16] Huang, H., Xie, H.B., Guo, J.Y., Chen, H.J. (2012). Ant colony optimization-based feature selection method for surface electromyography signals classification. Computers in Biology and Medicine, 42, 30-38.

[17] Hakonen, M., Piitulainen, H., Visala, A. (2015). Current state of digital signal processing in myoelectric interfaces and related applications. Biomedical Signal Processing and Control, 18, 334-359.

[18] Kilby, J., Prasad, K., Mawston, G. (2016). Multichannel surface electromyography electrodes: A review. IEEE Sensors Journal, 16, 5510-5519.

[19] Lucas, M.F., Gaufriau, A., Pascual, S., Doncarli, C., Farina, D. (2008). Multi-channel surface EMG classification using support vector machines and signalbased wavelet optimization. Biomedical Signal Processing and Control, 3, 169-174.

[20] Farina, D., do Nascimento, O.F., Lucas, M.F., Doncarli, C. (2007). Optimization of wavelets for classification of movement-related cortical potentials generated by variation of force-related parameters. Journal of Neuroscience Methods, 162, 357-363.

Received December 17, 2016. Accepted May 15, 2017. 